\title{
EchoGéo
}

39 | 2017

Ressources urbaines (1)

\section{Les inégalités d'accès aux ressources urbaines dans les franges périphériques de Belo Horizonte (Brésil) : quelles évolutions?}

\section{Eugênia Viana Cerqueira}

\section{(2) OpenEdition}

\section{Journals}

Electronic version

URL: https://journals.openedition.org/echogeo/14856

DOI: $10.4000 /$ echogeo.14856

ISSN: 1963-1197

Publisher

Pôle de recherche pour l'organisation et la diffusion de l'information géographique (CNRS UMR 8586)

Electronic reference

Eugênia Viana Cerqueira, "Les inégalités d'accès aux ressources urbaines dans les franges périphériques de Belo Horizonte (Brésil) : quelles évolutions ?", EchoGéo [Online], 39 | 2017, Online since 28 March 2017, connection on 10 August 2021. URL: http://journals.openedition.org/echogeo/ 14856 ; DOI: https://doi.org/10.4000/echogeo.14856

This text was automatically generated on 10 August 2021

EchoGéo est mis à disposition selon les termes de la licence Creative Commons Attribution - Pas d'Utilisation Commerciale - Pas de Modification 4.0 International (CC BY-NC-ND) 


\title{
Les inégalités d'accès aux ressources urbaines dans les franges périphériques de Belo Horizonte (Brésil) : quelles évolutions?
}

\author{
Eugênia Viana Cerqueira
}

1 Dans des métropoles de plus en plus étalées, les inégalités d'accès aux opportunités offertes par les espaces urbains sont de plus en plus prononcées (Caubel, 2006). L'injonction à la mobilité s'impose comme une condition majeure d'insertion dans la société actuelle, ce qui contribue à écarter une partie non négligeable des populations (Bacqué et Fol., 2007). Ces inégalités tendent à s'accentuer encore plus lorsque l'on s'éloigne du centre des agglomérations (Jouffy et al., 2015) : les espaces périphériques, moins desservis en services et équipements, reposent de plus en plus sur une mobilité automobile qui construit une offre à la carte des équipements et services urbains (Chalas, 2000). De fait, la capacité d'atteindre une ressource donnée devient une entrée fondamentale de la problématique des inégalités sociales: elle renvoie non seulement à la localisation des équipements dans la ville, mais aussi au potentiel des populations à se déplacer en fonction de leurs caractéristiques individuelles.

2 Ces questionnements s'appliquent davantage aux métropoles latino-américaines, dans lesquelles les modèles d'organisation spatiale entraînent un accroissement important des mobilités. L'expansion et la diversification de l'offre résidentielle dans les périphéries permettent aux individus de déménager plus loin, ce qui engendre une restructuration de leurs pratiques de mobilité (Dureau et al., 2015). Si les villes des Suds ont été longtemps marquées par de nombreuses inégalités dans les conditions de déplacement des populations, on pourrait s'interroger sur les impacts des nouveaux modes d'habiter les marges de villes sur la capacité d'accès des ménages aux ressources urbaines: il importe de considérer que cet accès peut évoluer sous l'effet de 
changements d'accessibilité spatiale, de l'offre de mobilité et de changements de localisation des populations et des ressources (Dureau et al., 2011).

3 Nous chercherons à analyser les inégalités d'accès aux ressources urbaines dans les franges métropolitaines de Belo Horizonte (Brésil). Le développement de cette métropole a été marqué par un modèle d'urbanisation fortement ségrégatif. On y constate des fortes inégalités de mobilité au niveau métropolitain: le modèle monocentrique de concentration d'équipements et d'activités a produit une forte dépendance du centre-ville. Depuis les années 1990, on observe dans les franges métropolitaines de nombreuses transformations qui passent à la fois par l'arrivée de nouvelles catégories de population et par une densification en termes d'équipements (Andrade et al., 2015). On peut donc s'interroger sur les impacts de ces évolutions récentes sur les comportements de mobilité des habitants. La diversité croissante des franges périphériques de Belo Horizonte, en matière de populations et d'équipements, contribue-t-elle à nuancer ou à renforcer les inégalités d'accès aux ressources urbaines?

\section{La Région Métropolitaine de Belo Horizonte : de nouvelles « périphérisations » ou de vieilles ségrégations?}

4 La croissance de la ségrégation et le renforcement des inégalités urbaines se font ressentir non seulement sur l'accès au marché de l'emploi, mais aussi sur l'ensemble des aménités urbaines. Comme le montre la propre définition du mot "ressources », ce concept renvoie aux "possibilités offertes par quelque chose». Le recours aux ressources dans l'espace urbain est exprimé par la relation entre un besoin et les activités disponibles, en particulier les biens et services dont les individus bénéficient au quotidien.

Dans le cadre de cet article, nous nous concentrons plus précisément sur les mobilités pour les achats, démarches, santé et loisirs. Ce choix renvoie aux travaux de David Caubel, qui définit un «panier de biens » par l'intermédiaire de «l'interprétation des motifs de déplacements les plus récurrents - structure a minima - des individus, quelle que soit leur appartenance à des groupes sociaux, leur niveau de vie ou leur position sociale.» (Caubel, 2006, p. 179).

6 La métropole de Belo Horizonte, capitale de l'État de Minas Gerais, encadre une région métropolitaine de plus de 5,7 millions d'habitants (IBGE, 2014) et occupe près de $9400 \mathrm{~km}^{2}$. La croissance métropolitaine s'est classiquement opérée selon une structure centre-périphérie couplée à des logiques de ségrégation. Dans les dernières décennies, la Région Métropolitaine de Belo Horizonte (RMBH) a suivi les tendances des autres métropoles brésiliennes, dans lesquelles les taux de croissance démographique des villes de la région métropolitaine dépassent ceux du pôle-centre. Ainsi, Andrade et al. (2015) soulignent que la RMBH est animée de "nouvelles périphérisations ", qui passent par la consolidation des périphéries traditionnelles, ainsi que par des processus simultanés de décentralisation et de fragmentation. Mendonça (2002) démontre que depuis les années 1990 l'expansion des fronts du marché immobilier a engendré une plus grande diversité des espaces périphériques dans la RMBH. 
Illustration 1 Carte de localisation : la Région Métropolitaine de Belo Horizonte (RMBH)

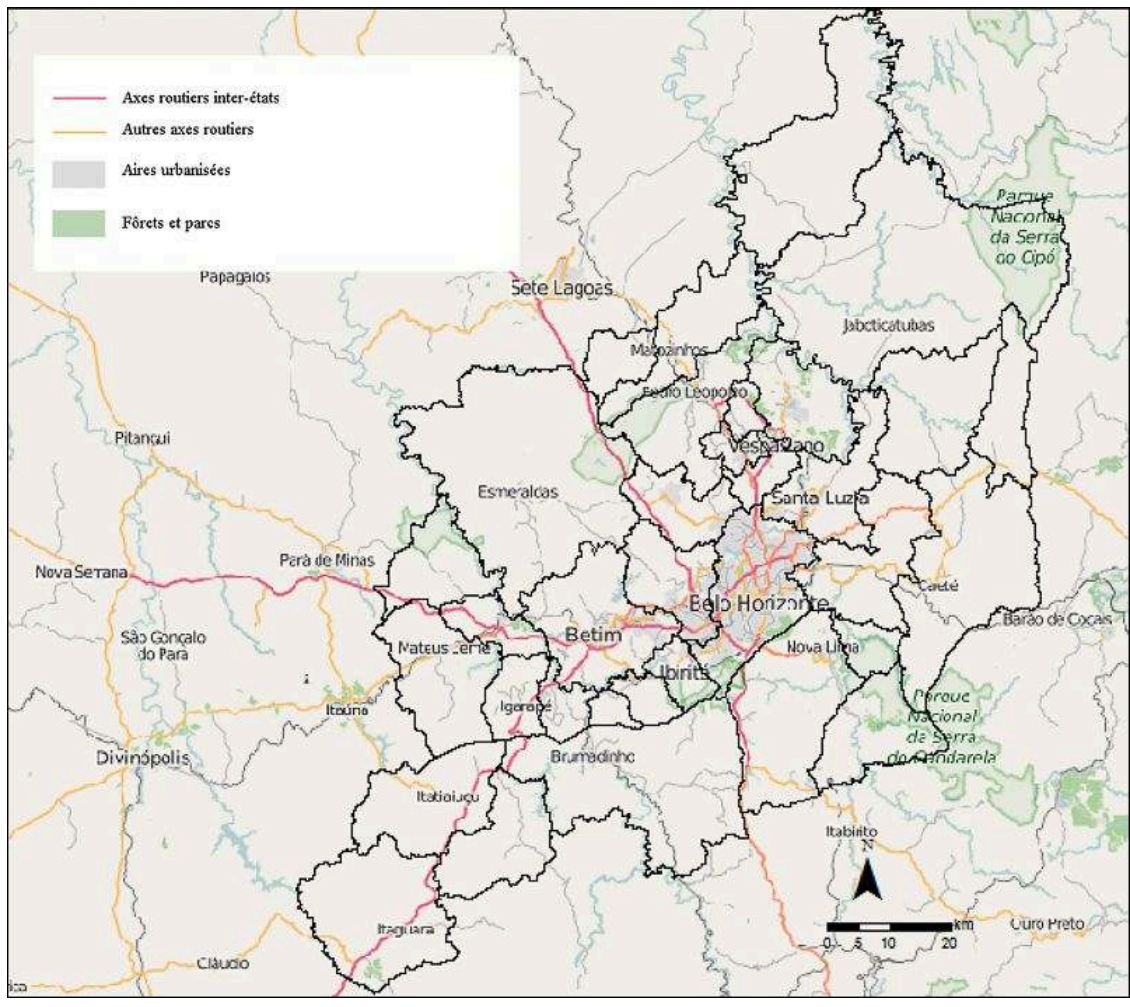

Sources : OpenStreetMap, PDDI RMBH ; réalisation : E. Viana Cerqueira, 2016.

7 Le secteur Ouest de la RMBH (municipalités de Contagem, Betim, Ibirité, Sarzedo et Mário Campos) se caractérise par l'ancienneté de son urbanisation, qui date des années 1940. L'expansion de ces périphéries a été fortement liée au développement de «l'axe industriel» depuis l'installation de grandes multinationales comme FIAT dans les années 1970. Depuis les années 2000, ces territoires sont devenus l'un des principaux axes d'expansion du pôle-centre, accueillant des nouveaux investissements immobiliers destinés notamment aux classes moyennes et s'imposant comme de nouvelles opportunités d'accession à la propriété en dehors du centre-ville.

Le secteur Nord de la RMBH (dont les principales municipalités sont Ribeirão das Neves, Santa Luzia, Vespasiano, São José da Lapa et Esmeraldas) a également connu de nombreuses transformations récentes. Ces espaces se sont développés dans les années 1970 avec la migration rurale des populations modestes, qui ne pouvant pas se loger au centre-ville, se sont installées dans ces périphéries populaires. Cette expansion a été fortement marquée par les lotissements populaires et par l'habitat informel. Plus récemment, le secteur est devenu la cible d'une importante vague d'investissements publics et privés avec l'installation de la Cité Administrative de l'État de Minas Gerais. C'est également dans ces espaces que l'on compte la production la plus importante de logements sociaux de la RMBH. Selon le dernier recensement de l'IBGE à Ribeirão das Neves, l'une des municipalités les plus pauvres de ce secteur, le taux de motorisation était de $35 \%$ en 2010 et la part d'individus ayant un diplôme supérieur était de seulement $3 \%$. Dans ce secteur, à l'exception de la municipalité de Lagoa Santa, marquée par la présence de lotissements fermés de haut standing, le taux d'individus diplômés du supérieur est inférieur à la moyenne des franges périphériques. 
9 Dans le cadre d'une forte polarisation sociale, à partir des années 1990, on a assisté à la consolidation du secteur Sud de la RMBH comme épicentre du développement des lotissements fermés et de la production immobilière destinée aux classes aisées. Les municipalités de Nova Lima et, dans une moindre mesure, Brumadinho et Rio Acima deviennent un secteur d'expansion du pôle-centre. Cette transformation, accompagnée de l'installation de nouvelles activités commerciales, de services et de loisirs (Mendonça et al., 2004), et d'une forte valorisation foncière contribué à attirer des activités spécialisées destinées aux populations riches habitant dans ces périphéries. En 2010, Nova Lima comptait $55 \%$ de ménages motorisés et $20 \%$ d'individus ayant un diplôme d'enseignement supérieur, soit 14 points de plus que la moyenne des municipalités périphériques.

10 Il convient de noter que, malgré les nombreuses transformations produites récemment dans les espaces périphériques de la RMBH, on y observe toujours des dynamiques qui renforcent le modèle traditionnel d'occupation de ces territoires. Ainsi, on remarque des processus et des configurations qui dessinent des logiques plus complexes de peuplement, mais aussi de distribution des aménités, équipements et services. On fait l'hypothèse que cette maturation des dynamiques périphériques, entraînerait une inflexion dans l'accès aux équipements, au-delà des oppositions centre-périphérie. On pourrait, ainsi, supposer qu'une décentralisation des équipements et l'évolution des comportements de mobilité pourraient contribuer à l'amélioration de l'accès des habitants des périphéries aux ressources urbaines. Tout d'abord, nous identifierons l'accès potentiel aux ressources urbaines en observant leur distribution dans la RMBH. Ensuite, nous analyserons le potentiel d'accès des habitants des franges périphériques de la RMBH aux équipements.

\section{Sources et méthodes : les mobilités vers les ressources urbaines.}

11 Dans un premier temps, on cherche à mesurer la distribution des ressources dans la RMBH en calculant la densité relative d'équipements par secteur. On met en œuvre la base de données $\mathrm{CEMIG}^{1}$ qui précise, par catégorie, le nombre d'abonnés au réseau électrique. À partir de ces informations, on peut distinguer les usagers selon trois catégories: commerciales, résidentielles et industrielles. Seule base fiable sur la localisation des équipements, on utilise ces informations, pour la catégorie des activités qualifiées comme "commerciales", afin de déterminer en première approximation l'ensemble du secteur des services qui constituent des ressources urbaines, aménités et services commerciaux. Une importante limite de cette méthode est l'absence de bases de données statistiques précisant la nature des équipements (activités de loisirs, santé, etc.), ce qui ne permet pas d'évaluer en détail la distribution des ressources dans la Région Métropolitaine de Belo Horizonte. Mais le terrain et les entretiens approfondis viennent renseigner utilement ces aspects.

12 Par ailleurs, on s'appuie sur l'enquête ménages-déplacements réalisée par la Fundação João Pinheiro en 2012, dont l'échantillonnage porte sur 13000 ménages, soit 44000 personnes $^{2}$. Les déplacements sont analysés en termes de lieux d'originedestination, mode de transport et motif de destination ${ }^{3}$ (commerces, démarches, santé et loisirs) ${ }^{4}$. Ainsi, on réalise un appariement entre les mobilités des ménages et la densité d'équipements. On travaille ainsi avec l'accès potentiel des populations, c'est-à- 
dire, le nombre de ressources auxquelles les individus peuvent potentiellement accéder en se déplaçant vers un endroit donné.

Illustration 2 - Modèle conceptuel des données

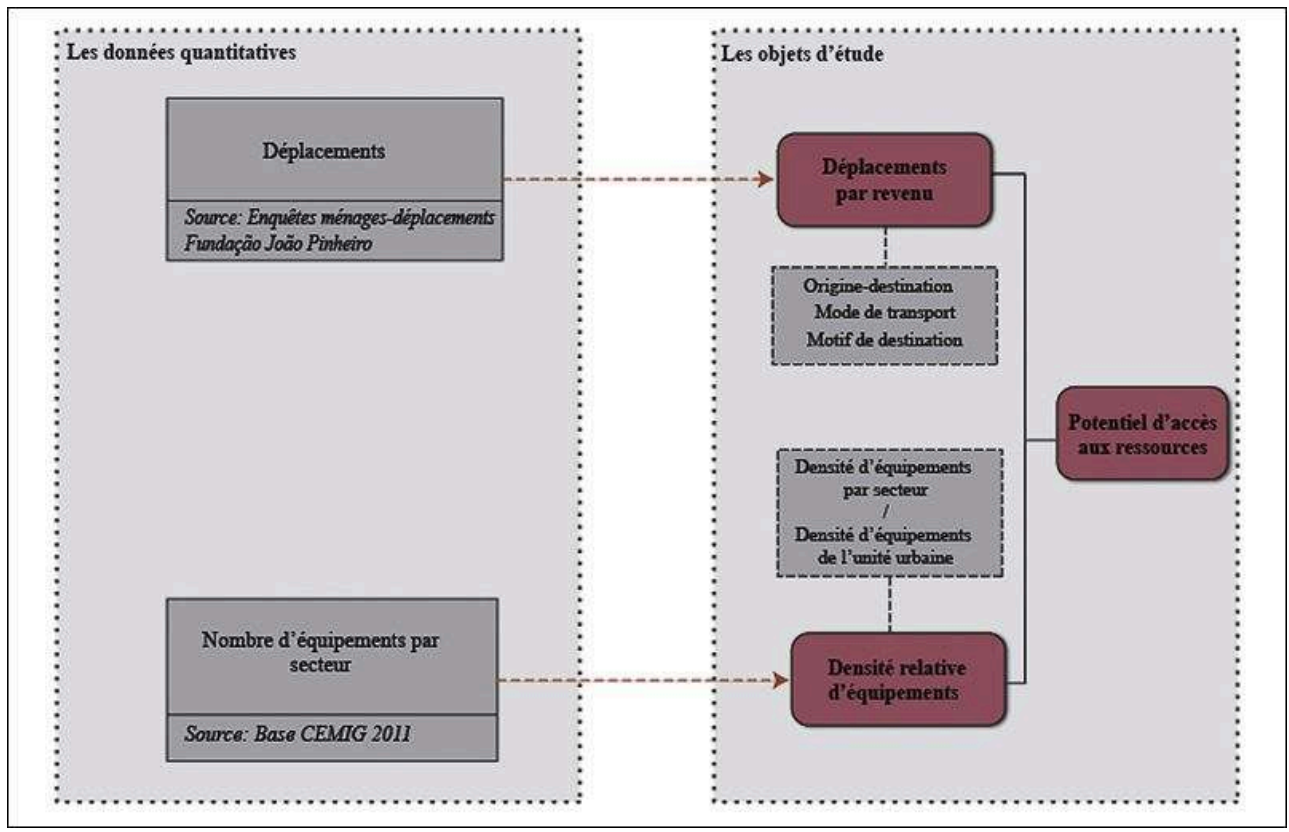

De plus, les enquêtes-ménages déplacements seules ne permettant pas de rendre compte des spécificités liées aux expériences quotidiennes individuelles, une campagne d'entretiens semi-directifs exploratoires a été menée auprès d'une douzaine de ménages $^{5}$. Il s'agit de mettre l'accent sur les différences des pratiques de mobilité et des représentations en fonction des contextes sociaux, résidentiels, professionnels. L'analyse des entretiens vient ainsi éclairer et nuancer les rapports que les habitants entretiennent avec l'espace (Berroir et al., 2015). Cette approche permet de saisir les mécanismes et stratégies déployées par les habitants afin d'accéder aux opportunités offertes par la ville, comme la mobilisation des ressources de proximité et des réseaux sociaux.

\section{Évaluation du potentiel d'accès aux ressources urbaines dans la Région Métropolitaine de Belo Horizonte}

Afin d'évaluer la manière dont les ressources sont distribuées dans la RMBH, on estime la densité relative des ressources urbaines, calculée à partir du rapport entre la densité d'équipements dans une unité spatiale donnée et celle de l'ensemble de l'unité urbaine (Le Goix et Guérois, 2000). Cette méthode permet de comparer les franges métropolitaines moins desservies et les espaces centraux, toutes choses égales par ailleurs quant à la densité moyenne de la région urbaine. On obtient alors trois catégories d'accès potentiel : « faible », « moyen », « fort » ${ }^{6}$. 


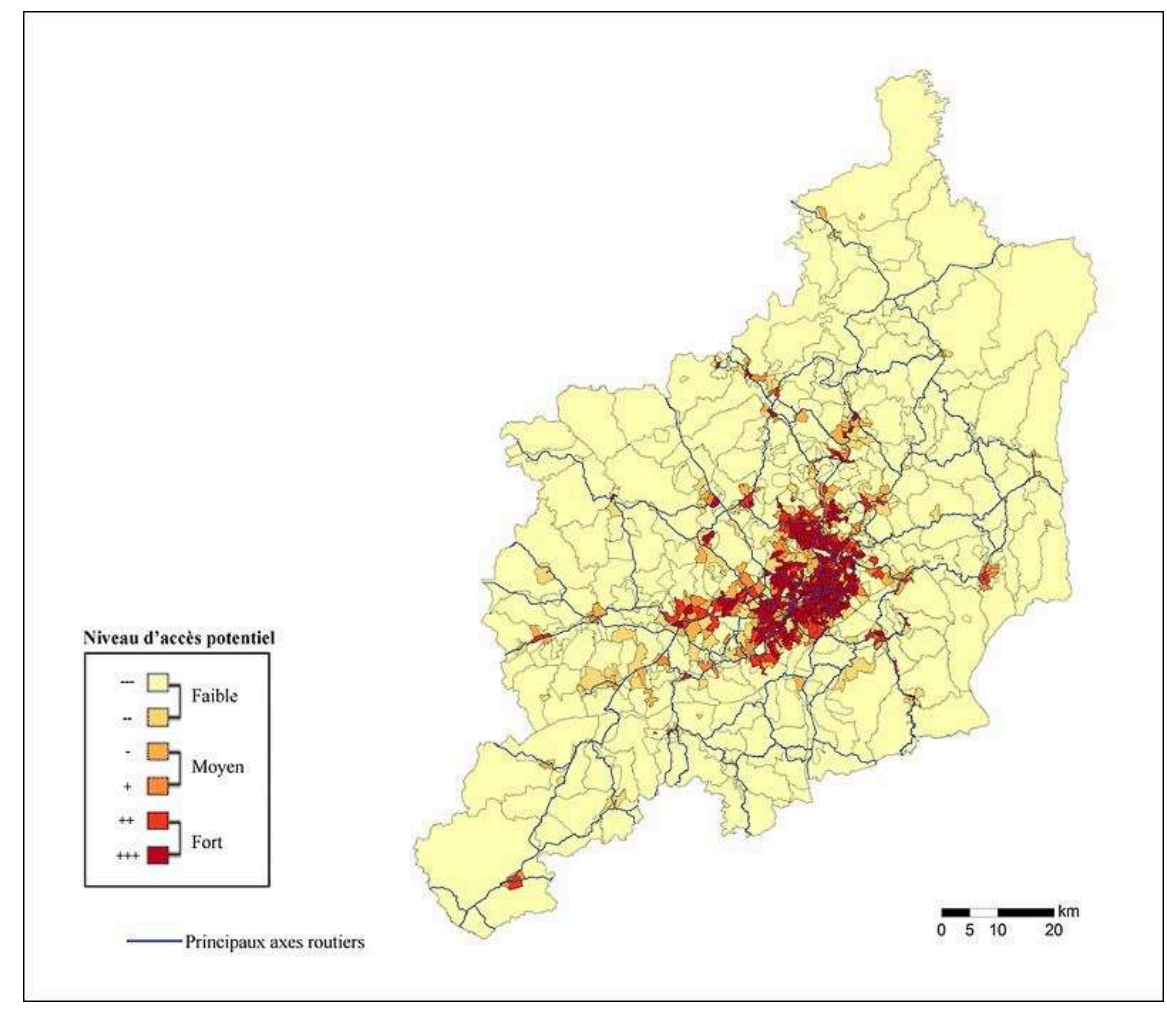

Source : Base CEMIG PDDI, 2009 ; réalisation : E. Viana Cerqueira, 2016.

15 Les secteurs identifiés comme « fort » se situent notamment dans le pôle-centre et dans les villes de Betim et Contagem, à l'Ouest de la métropole. On observe également des centralités périphériques avec une forte densité de ressources au sein des villes de Nova Lima, Ribeirão das Neves et Sabará. Par ailleurs, la densité relative de ressources tend à être plus élevée le long des principaux axes routiers métropolitains : BR-040, Avenida Amazonas.

En contemplant ainsi la densité relative des secteurs de destination de chaque déplacement, on évalue le potentiel d'accès des individus aux ressources en fonction non seulement de leurs caractéristiques individuelles, mais aussi de la structure urbaine. On fait l'hypothèse que lorsque les individus se déplacent vers des secteurs bien équipés ils disposent d'un accès potentiel à un nombre d'équipements plus important. Il s'agit d'analyser, par la suite, la manière dont les périphéries se structureraient selon un modèle de plus en plus polycentrique, caractérisé par un desserrement croissant des équipements et une inflexion des comportements de mobilité.

\section{Les inégalités d'accès aux ressources urbaines : une approche par les revenus}

17 La seconde partie de cette recherche consiste à analyser les inégalités d'accès à partir de l'enquête ménages-déplacements réalisée en 2012. Le revenu a été choisi comme principal critère d'évaluation, car « la pauvreté en ressources économiques a un effet propre sur la mobilité quotidienne » (Jouffe et al., 2015). Ces revenus sont indiqués par 
référence au montant du salaire minimum $(\mathrm{SM})^{7}$ et ont été regroupés en cinq tranches (de moins de 1 à plus de 10 fois le salaire minimum). Dans le contexte brésilien, comme dans beaucoup d'autres contextes où l'écart entre les revenus s'est considérablement accru dans les dernières années, ceux-ci semblent s'imposer comme de meilleurs indicateurs des inégalités sociales que les catégories socio-professionnelles. Cette analyse pourrait encore être approfondie en fonction d'autres caractéristiques individuelles comme le niveau d'étude, l'âge, le sexe.

\section{Un accès potentiel toujours privilégié pour les populations aisées}

Pour toutes les catégories, plus de $80 \%$ des déplacements sont réalisés vers des secteurs à moyen ou fort accès potentiel. Ces résultats indiquent toujours une dépendance des secteurs très bien desservis à Belo Horizonte et à Contagem, qui représentent $33 \%$ des destinations des déplacements observés.

Les populations les plus aisées sont celles qui témoignent d'un accès potentiel plus important, se déplaçant plus vers des secteurs à forte accessibilité que les populations les plus pauvres (on constate un écart de 25 points entre ces catégories, cf. illustration 3). Ces dernières tendraient à limiter la portée de leurs déplacements hors travail, ce qui se traduit par un moindre accès potentiel des ménages habitant dans des territoires moins équipés.

Illustration 4 - Niveau d'accès potentiel des secteurs de destination en fonction des revenus

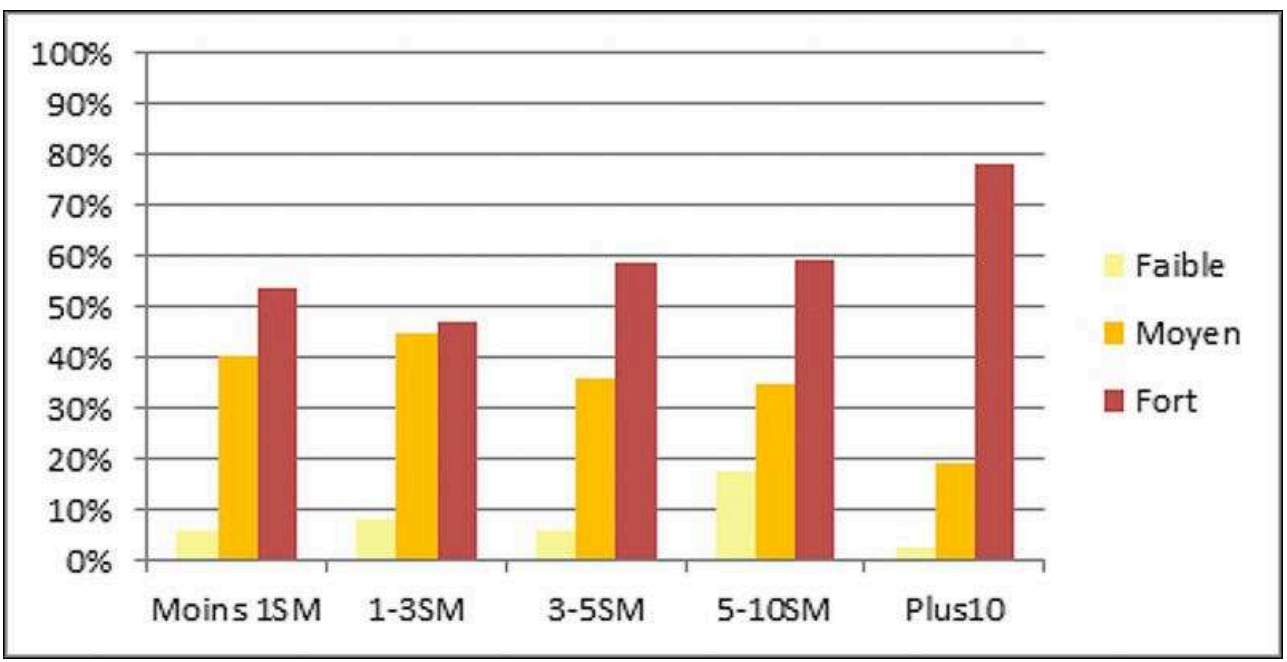

Source : EMD 2012 - Fundação João Pinheiro ; réalisation : E. Viana Cerqueira, 2016.

\section{Le gain d'accès potentiel : le poids de la mobilité automobile}

Il convient également d'évaluer si les opportunités d'accès aux ressources augmentent ou diminuent lorsque les individus réalisent un déplacement. Vallée et al. (2015) proposent une approche permettant d'observer le " gain d'accessibilité ${ }^{8}$ induit par les mobilités quotidiennes à partir des différents lieux d'activité des individus : «L'idée est 
ainsi de comparer les "champs des possibles" des différents groupes sociaux (Wenglenski, 2004) et d'évaluer les gains d'accessibilité induits par la mobilité quotidienne » (Vallée et al., 2015, p. 2)

Illustration 5 - Le gain d'accès potentiel par mode de transport en fonction des revenus

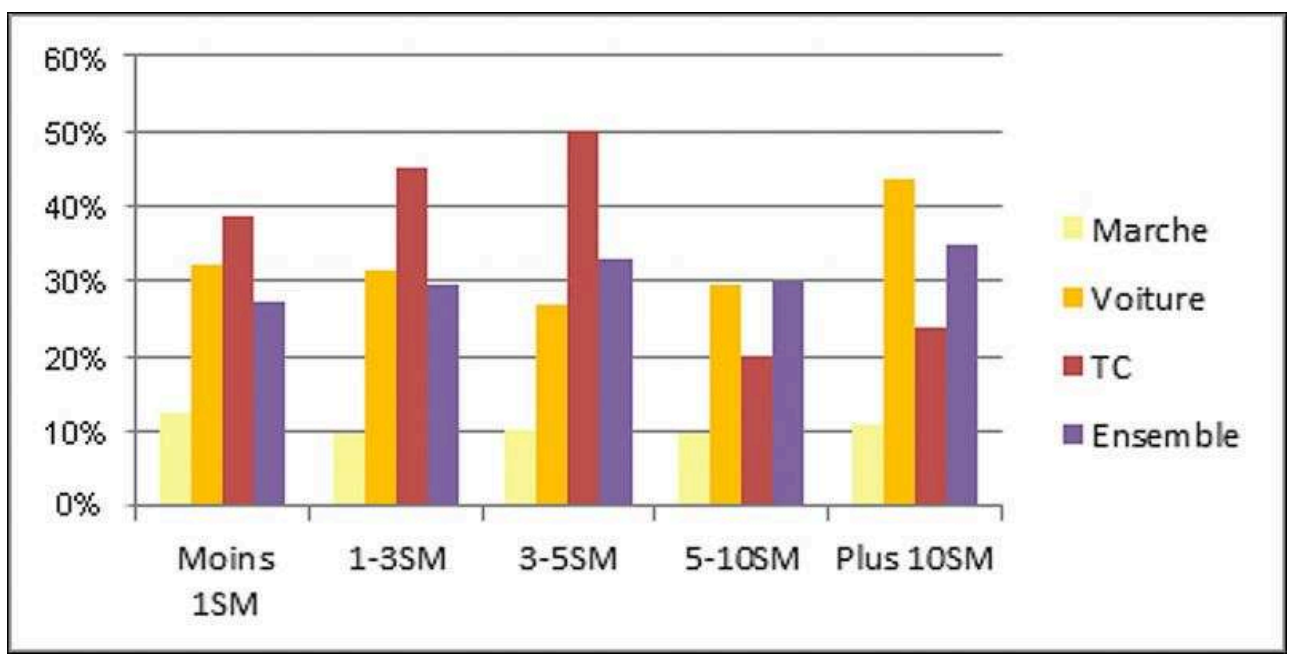

Source : EMD 2012 - Fundação João Pinheiro ; réalisation : E. Viana Cerqueira, 2016.

21 Un résultat important distingue les ménages : les déplacements en voiture engendrent une augmentation du potentiel d'accès beaucoup plus élevée pour les populations aisées. En revanche les ménages modestes pour leur part sont confrontés à des gains d'accès beaucoup plus significatifs lorsqu'ils se déplacent en transports en commun. Ces résultats traduisent le faible accès à la voiture de ces ménages et leur dépendance aux transports en commun pour se déplacer et pour accéder à des zones mieux équipées en commerces et services (66\% des populations les plus pauvres ayant à se déplacer ne disposent pas d'une voiture). On peut faire l'hypothèse que, même motorisés, ces ménages chercheraient plutôt à limiter la portée de leurs déplacements en voiture en utilisant les transports en commun pour parcourir des distances plus longues et pour accéder aux espaces les mieux desservis.

En analysant la courbe d'accessibilité par revenus (illustration 6), on note que les populations aisées sont celles qui atteignent potentiellement le plus grand nombre de ressources en voiture : $80 \%$ de leurs déplacements pour les activités observées ont une durée inférieure à 30 minutes. À l'opposé, les populations les plus pauvres semblent avoir une accessibilité beaucoup plus limitée lorsqu'elles sont motorisées. Ces ménages réalisent la moitié des déplacements que les classes aisées effectuent en 30 minutes. 
Illustration 6 - L'accès aux ressources en voiture en fonction des revenus

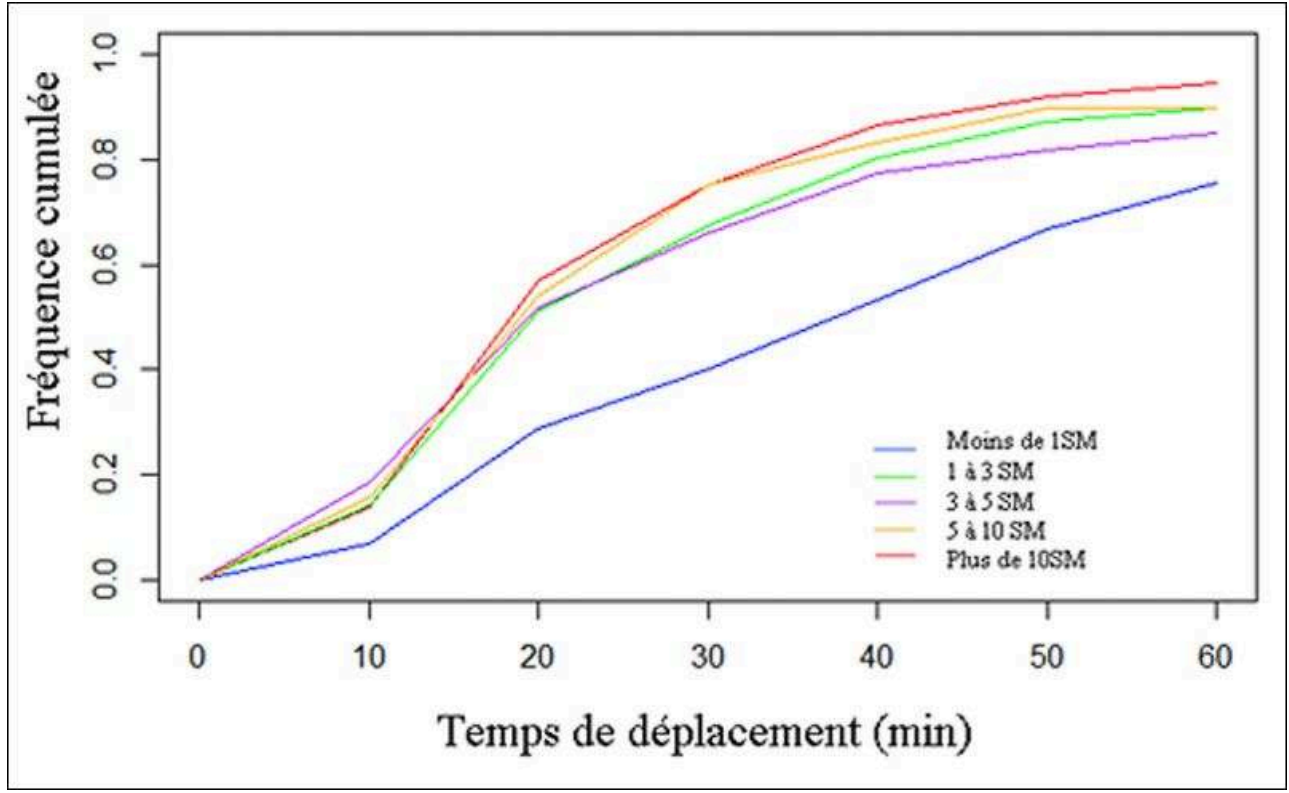

Source: EMD 2012 - Fundação João Pinheiro ; réalisation : E. Viana Cerqueira, 2016.

\section{Les inégalités d'accès par motif de destination : des comportements sociaux marqués}

L'analyse des déplacements et des inégalités d'accès aux équipements par motif de destination révèle des comportements sociaux marqués selon le type d'activité réalisée. Pour l'échantillon analysé les activités commerciales et de loisirs représentent respectivement $22 \%$ et $43 \%$ des déplacements hors travail et études, alors que les démarches et la santé représentent pour leur part $17 \%$ et $19 \%$ des déplacements.

Illustration 7 -- Gain d'accès par motif de destination en fonction des revenus

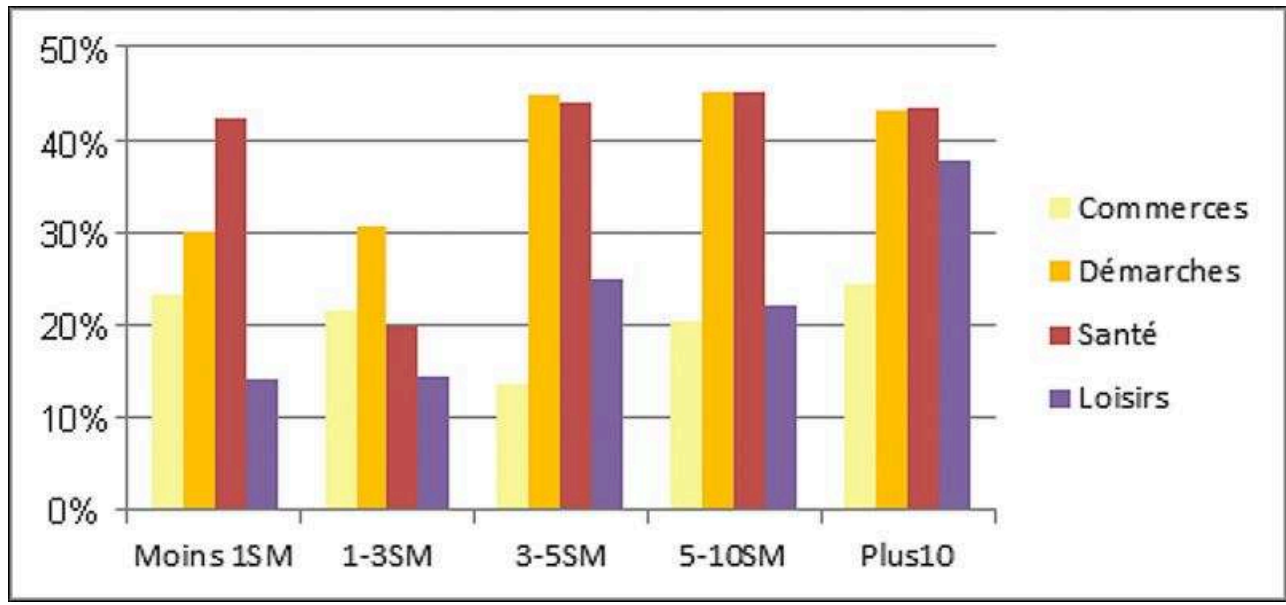

Source : EMD 2012 - Fundação João Pinheiro; réalisation : E. Viana Cerqueira, 2016. 


\section{Les achats: des déplacements limités pour les ménages modestes?} les plus faibles gains d'accès. Dans la RMBH, plus de $60 \%$ des déplacements des populations les plus pauvres pour les commerces sont effectués à pied, tandis qu'une majorité de $89 \%$ des populations aisées utilisent des moyens motorisés pour ces pratiques. Des travaux récents (Morel-Brochet et Motte-Baumvol, 2010) soulignent que parmi les ménages modestes les déplacements pour les achats tendent à être plus rares et plus courts. Pour la plupart des populations modestes enquêtées, l'absence ou l'accès limité à une voiture sont souvent évoqués pour expliquer le recours à la proximité et à la marche à pied. Certains profitent des déplacements professionnels pour réaliser leurs achats, notamment alimentaires, ainsi que le précisent les entretiens :

"Pour les achats, c'est plutôt près chez moi. On a tout ce qu'on a au centre, des magasins, des supermarchés. Je vais toujours à pied au supermarché, c'est près de chez moi. J'arrive du boulot en bus et puis je vais directement faire mes courses» (Femme, 55 ans, 1 à 3 SM, Ribeirão das Neves).

Par ailleurs, les données disponibles (enquête ménages-déplacement de 2012) ne permettent pas tout à fait de repérer les changements très récents qui pourraient encore renforcer les inégalités illustrées ci-dessus. C'est le cas, par exemple, des franges métropolitaines situées au Sud de la métropole, qui ont connu un développement urbain très important dans la période récente. L'accroissement significatif de l'offre de commerces et de services a permis de favoriser l'accès de ces populations aux équipements.

«Moi j'habite dans un condomínio à Nova Lima. Aujourd'hui il y a tous les types d'activité à côté, mais il y a 25 ans il fallait tout faire au centre-ville. Aujourd'hui il y tout à côté de chez moi : pharmacie, boulangerie, coiffeur. Je n'ai plus besoin de venir au centre-ville si je ne veux pas » (Femme, 68 ans, plus de $10 \mathrm{SM}$ ).

Les entretiens mettent en évidence que la majorité des enquêtés considèrent leurs quartiers bien desservis en matière d'équipements commerciaux. À l'exception de certains types spécifiques de commerce (meubles, bricolage, etc.) les pratiques semblent s'inscrire plutôt à l'échelle locale. L'illustration 8 révèle que la grande majorité des catégories accèdent à près de $60 \%$ des équipements d'achat en moins de 20 minutes. Pourtant, les populations aisées disposent dans l'ensemble d'un meilleur accès à ce type d'équipement : $80 \%$ des déplacements pour les achats ont une durée inférieure à 30 minutes. 
Illustration 8 - L'accès aux équipements d'achat en fonction des revenus

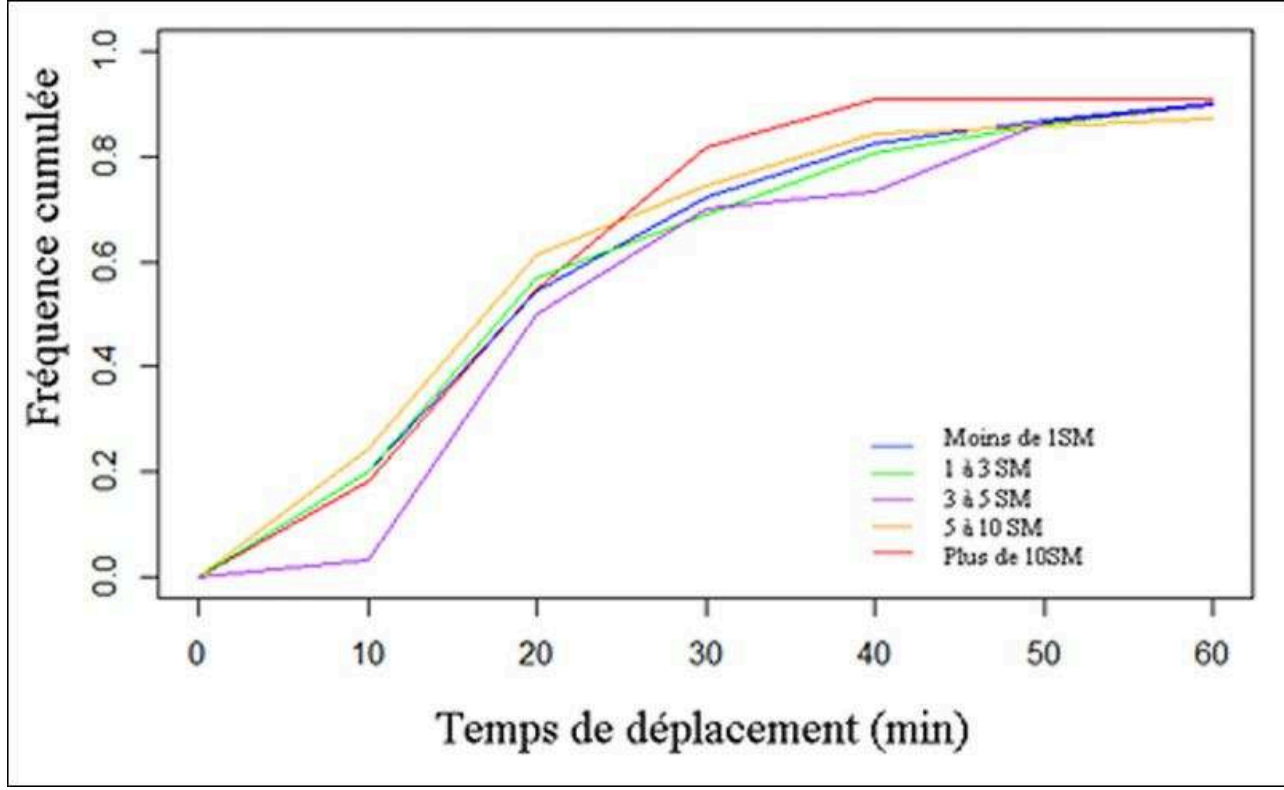

Source : EMD 2012 - Fundação João Pinheiro ; réalisation : E. Viana Cerqueira, 2016.

\section{Les démarches et la santé : une dépendance toujours importante du centre}

Les déplacements pour la santé et pour les démarches sont ceux qui occasionnent les plus forts gains d'accès potentiel pour toutes les catégories. Ces équipements tendent à se concentrer dans les secteurs les plus denses et mieux desservis en ressources : $45 \%$ et $35 \%$ des déplacements pour la santé et les démarches se font respectivement vers Belo Horizonte et Contagem. Si à première vue ces pratiques semblent être assez homogènes entre les catégories sociales, les entretiens permettent de tracer de nettes distinctions dans les mécanismes et tactiques de mobilité qui déterminent leur accès aux ressources urbaines.

En effet, les ménages modestes travaillant dans des secteurs bien équipés cherchent plutôt à optimiser leurs déplacements pour les démarches et services à partir de leurs mobilités professionnelles. Ces populations, souvent contraintes à subir des longues navettes domicile-travail, mobilisent des stratégies afin de pouvoir accéder aux différentes ressources durant leurs heures de travail. Ici, la mobilité depuis les lieux d'activité intervient comme un levier pour améliorer la capacité de maîtrise des populations modestes.

31 "Pour tout ce qui est banque, avocat, tout ce qui est administratif, je préfere de venir au centre, je vais ici au centre-ville. Je travaille ici, donc je profite du déplacement, parce que quand j'arrive du boulot tout est déjà fermé. Et aussi j'ai l'impression que les choses sont plus rapides à Belo Horizonte » (Femme, 47 ans, 1 à 3 SM, Betim).

Par ailleurs, la rationalisation de la localisation des activités de santé passe par l'implantation des grands pôles de concentration d'équipements de santé qui desservent population plus nombreuse, mais sont globalement moins accessibles (Lucas, 2006). Parmi les ménages modestes, $42 \%$ des déplacements pour la 
santé s'effectuent en transport collectif et constituent des obstacles en termes de mobilité.

"Je suis transplanté donc il faut que j'aille chez le médecin une fois par semaine. Tout ce dont j'ai besoin pour la santé, il faut venir au centre-ville, parce qu'ici c'est chaotique. Avant je prenais le car de la mairie et il fallait me lever à 4 h30 du matin pour arriver au centre vers $7 \mathrm{~h} 30$. Aujourd'hui je prends le bus parce que c'est plus rapide » (Homme, 59 ans, 1 à 3 SM, Betim).

Les populations les plus modestes ont un accès plus limité aux équipements de santé, en raison des longues distances et de la durée des déplacements (illustration 9). Or, moins de $40 \%$ des mobilités des pauvres pour la santé se réalisent en moins de 30 minutes. À l'opposé, les ménages aisés semblent avoir un accès beaucoup plus efficace à ce type d'équipement.

Illustration 9 - L'accès aux équipements de santé en fonction des revenus

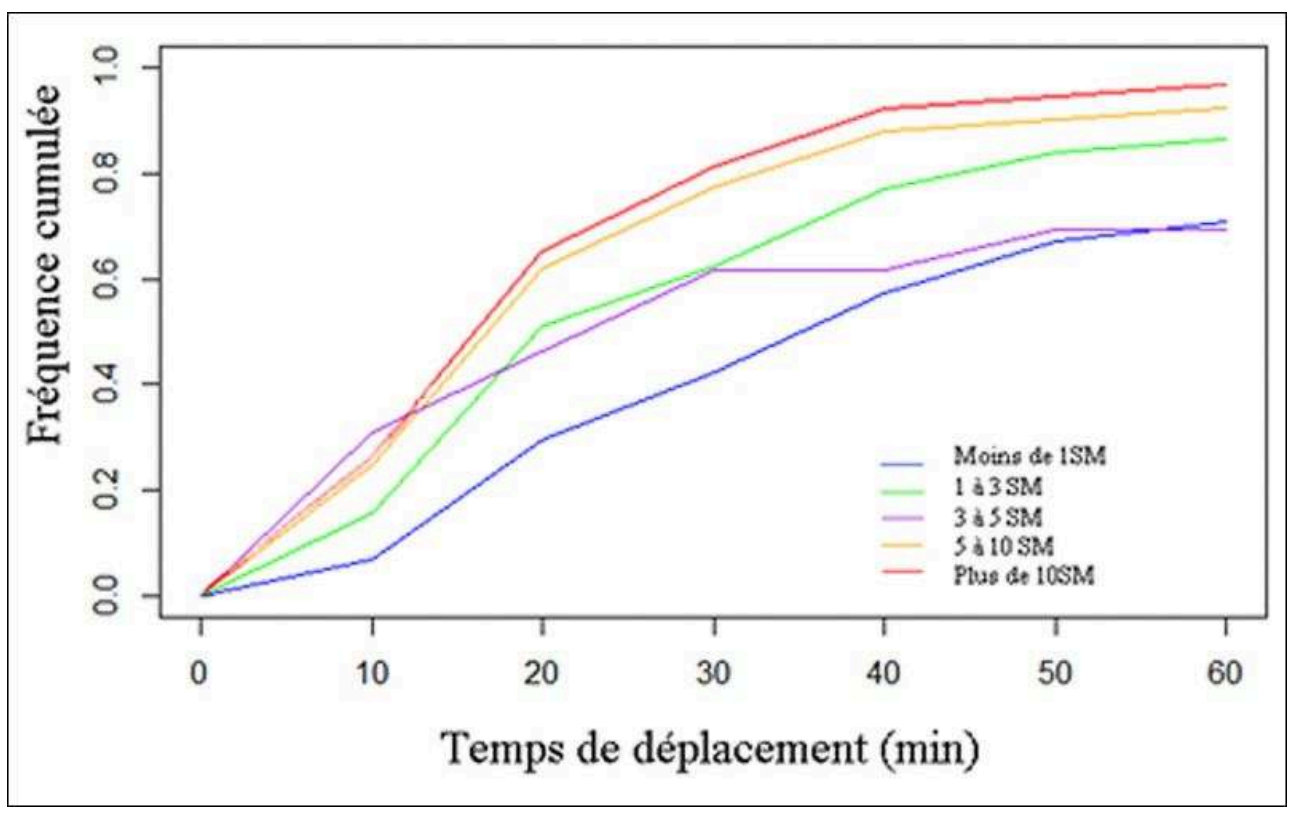

Source : EMD 2012 - Fundação João Pinheiro ; réalisation : E. Viana Cerqueira, 2016.

Les pratiques des populations aisées sont souvent justifiées par leurs choix individuels. On constate que, même s'ils disposent d'une offre d'équipements de santé à l'échelle locale, ce n'est pas la proximité du domicile qui oriente ce type de mobilité. L'accent est mis sur d'autres facteurs comme la «qualité du service» et les «habitudes" pour justifier leurs pratiques de santé.

"Je vais chez le médecin plutôt au centre-ville parce que j'ai gardé mes habitudes. Mes médecins ils sont tous au centre-ville, sauf ceux qui sont à l'hôpital Biocor, à côté de Nova Lima. " (Femme, 68 ans, plus de 10 SM, Nova Lima).

\section{Les loisirs : faire avec ce qu'il y a à proximité}

Les mobilités pour les loisirs sont celles qui engendrent des inégalités les plus marquées en termes de gain d'accessibilité. On constate que les ménages aisés bénéficient d'une augmentation de l'accès potentiel près de trois fois plus importante que les populations modestes. Ces pratiques ont également un poids plus important chez les plus riches: 
elles représentent plus de $50 \%$ des leurs déplacements hors travail contre $40 \%$ chez les individus gagnant moins de $1 \mathrm{SM}$.

Les catégories les moins aisées tendraient à s'orienter davantage vers les activités du domicile, car leur capital ne leur permettrait pas de dépenser aussi fréquemment que les classes aisées (Rougé, 2007). La faible mobilité et le manque de moyens financiers se traduisent alors par des limitations dans les pratiques de loisirs dont le choix n'est pas très large, spécialement dans les périphéries moins desservies (Jouffe et al., 2015). La plupart des enquêtés des ménages modestes sortent peu pour les activités de loisirs et, quand ils le font, ils restent inscrits dans une logique de proximité : plus de $55 \%$ des déplacements sont réalisés à pied.

"Ah moi je n'ai pas d'activité de loisir, je n'ai pas le temps. Je vais à l'église une fois par semaine, c'est à côté de chez moi. Quand je sors du boulot je n'ai pas envie de retourner au centre-ville pour aller au cinéma, ce genre de choses. Près de chez moi je vais à la piscine de temps en temps... ben, tous les 36 du mois » (Femme, 49 ans, moins de 1 SM, Ribeirão das Neves)

Parmi les ménages motorisés à bas revenu, les déplacements en voiture restent souvent restreints aux sorties de loisir, car ils ne disposent pas des moyens financiers pour entretenir le véhicule pour une utilisation quotidienne. Le manque de ressources économiques les conduit par conséquent à déployer des stratégies afin de pouvoir accéder à ces équipements.

41 "Pour travailler je prends la moto et pour les loisirs, pour aller au cinéma avec mes enfants, par exemple, je prends la voiture. La voiture c'est plutôt le week-end. Il faut toujours faire des économies, n'est pas? » (Homme, 44 ans, 3 à 5 SM, Nova Lima).

Les ménages aisés, pour leur part, semblent s'inscrire dans une logique de choix « à la carte » en ce qui concerne les activités de loisir. Pour ces populations, près de $80 \%$ de déplacements pour atteindre ce type d'équipements se réalisent en voiture. Ces déplacements sont liés davantage aux choix individuels des ménages, qui choisissent de se déplacer plus loin pour atteindre un des équipements spécifiques (comme des sorties au cinéma, musée etc.), souvent situés en centre-ville.

43 Par ailleurs, ces populations disposent le plus souvent d'une importante offre de loisirs à proximité de leur domicile. C'est le cas, par exemple, des ménages habitant à Nova Lima. Le développement récent de ce secteur a contribué à attirer une multitude d'équipements. Par ailleurs, on y trouve également une gamme d'activités de loisir (notamment de sport) à l'intérieur des lotissements fermés. Ici, le repli au domicile constitue un choix et non pas une contrainte comme observé chez les ménages modestes.

44 "Je ne sors pas beaucoup pour les loisirs, je suis assez casanière. Ben, j'ai tout chez moi : piscine, sauna, home theater. Je fais du jogging à l'intérieur du condomínium. C'est le style de vie que j'ai choisi et ma situation le permet »(Femme, 68 ans, plus de 10 SM, Nova Lima).

\section{Conclusion}

Les analyses et les premiers entretiens valident l'hypothèse selon laquelle les inégalités d'accès aux ressources urbaines sont toujours prononcées, malgré la diversité croissante des franges métropolitaines belorizontines. Si dans les dernières années les marges de la ville sont devenues des cibles majeures de l'industrie immobilière, ces 
évolutions ne semblent pas contribuer à la réduction des écarts d'accès entre les catégories sociales.

Les résultats mettent en évidence que les populations les plus aisées bénéficient toujours d'un accès à l'ensemble des équipements de la métropole plus important. Ces différentiations se cumulent avec de fortes inégalités de droits aux modes de déplacements. Même si les populations pauvres ont des gains d'accès plus significatifs en transport en commun, cela ne leur permet pas de compenser les écarts induits par la mobilité automobile.

Par ailleurs, l'analyse des déplacements par motif de destination fait apparaître des comportements sociaux marqués, ainsi que des tactiques, déployées par les ménages afin d'accéder aux équipements, différentes. Les pratiques des populations aisées se dessinent à partir de leurs choix individuels et de la mobilité automobile, tandis que celles des ménages modestes sont orientées par des contraintes comme l'accès limité à la voiture et la localisation des ressources elles-mêmes. Les entretiens qualitatifs permettent de mettre en évidence la diversité de comportements de mobilité observés et les principales difficultés rencontrées par les ménages dans l'accès aux équipements.

Ces résultats permettent de construire un agenda de recherche sur la manière de penser et d'aménager les espaces périphériques. Il semble que même si les espaces périphériques ont connu des évolutions en termes d'implantations d'équipements, les populations sont toujours contraintes de se déplacer vers des espaces très desservis pour accéder à certaines ressources. Il s'agit ainsi de penser les marges de la ville en tenant en compte de la maturation croissante de ces espaces comme des polarités potentielles capables de structurer les pratiques quotidiennes des habitants. La complexité des modes de vivre des franges métropolitaines invite à un changement de regard des franges métropolitaines et à une réorientation des politiques urbaines, notamment en termes d'articulation entre la récente redistribution des ressources et les opportunités d'accès à la ville.

\section{BIBLIOGRAPHY}

Andrade L., Mendonça J., Diniz A. , 2015. Belo Horizonte: transformações na ordem urbana. Rio de Janeiro, Letra Capital : Observatório das Metrópoles, 474 p.

Bacqué M., Fol S., 2007. Inequality regarding mobility: observations and policies. Swiss Journal of Sociology, 33, 1, p. 89-104.

Berroir S., Desjardins X., Fleury A., Queva C. (coor), 2015. Lieux et hauts lieux des densités intermédiaires. Rapport de recherche, Plan Urbanisme Construction et Architecture, $234 \mathrm{p}$.

Cary P., 2012. Les centres commerciaux à São Paulo. Espaces et sociétés, 2, 150, p. 107-127.

Caubel D., 2006. Politique de transports et accès à la ville ? Une méthode d'évaluation appliquée à

l'agglomération lyonnaise. Thèse de Doctorat, Lyon, Université Lumière Lyon 2.

Chalas Y., 2000. L'invention de la ville. Paris, Antrophos, 199 p. 
Dureau F., Goueset V., Le Roux G., Lulle T., 2011. Mutations urbaines et inégalités d'accès aux ressources de la ville : quelques enseignements d'une collecte biographique sur les mobilités à Bogotá (Colombie). Mobilités spatiales et ressources métropolitaines : l'accessibilité en questions, Grenoble, France.

Dureau F., Lulle T., Souchaud S., Contreras Y. (dir.), 2015. Mobilités et changement urbain à Bogotá, Santiago et São Paulo. Rennes, Presses Universitaires de Rennes, 440 p.

Guérois M., Le Goix R., 2000. La multipolarité dans les espaces métropolitains: Paris, Lyon, Marseille et Lille. In Mattei M.-F. et Pumain D. (dir), Données urbaines (Volume 3), p. 235-249.

Hubert J.-P., 2004. Mobilité urbaine, périurbaine, rurale en Belgique : où sont les différences ? Les Cahiers Scientifiques du Transport, 45, p. 83-100.

Jouffe Y., Caubel D., Fol S., Motte-Baumvol B., 2015. Faire face aux inégalités de mobilité. Cybergeo : European Journal of Geography.

Lucas K., 2006. Providing transport for social inclusion within a framework for environmental justice in the UK. Transportation Research Part A , 40, 10, p. 801-809.

Mendonça J., 2002. Segregação e mobilidade residencial na região metropolitana de Belo Horizonte. Thèse de Doctorat, Rio de Janeiro, Universidade Federal do Rio de Janeiro.

Mendonça J., Perpétuo I., Vargas M., 2004. A periferização da riqueza na metrópole belohorizontina: falsa hipótese? XI Seminário de Economia Mineira, Diamantina. Cedeplar UFMG, Belo Horizonte.

Morel-Brochet A., Motte-Baumvol B., 2010. Les périurbains : stratégies résidentielles, tactiques du quotidien et résistance des modes d'habiter. In M.H. Massot (dir.), Mobilités et modes de vie métropolitains, les intelligences du quotidien, Paris, L'œil d'or, p. 93-112.

Ribeiro L.-C., Moura R., Delgado P., Silva E. , 2012. Níveis de integração dos municípios brasileiros em RMs, RIDES e AUS à dinâmica da metropolização. Rio de Janeiro, Observatório das Metrópoles, 108 p.

Rougé L., 2007. Les « captifs » du périurbain. Voyage chez les ménages modestes installés en lointaine périphérie. In Capron G., Cortès G., Guetat H. (dir.), Liens et lieux de la mobilité, Belin, p. 129-144.

Vallée J., Commenges H., Perchoux C., Kestens Y., Chaix B., 2015. Mobilité quotidienne et accès potentiel aux ressources urbaines en île-de-France : quelles inégalités sociales ? In Mattei M.-F. et Pumain D. (dir), Données Urbaines (Volume 7), p. 87-95.

Wenglenski S., 2004. Une mesure des disparités sociales d'accessibilité au marché de l'emploi en Île-de-France. Revue d'Économie Régionale et Urbaine, 4, p. 539-558.

\section{NOTES}

1. Companhia Energética de Minas Gerais.

2. Nous observons toutes les municipalités de la RMBH à l'exception de Belo Horizonte et de la ville de Contagem. Compte tenu de la densité et du niveau d'intégration de cette ville à Belo Horizonte, cette dernière est considérée comme une extension du pôle-centre dans la typologie développée par l'Observatório das Metrópoles (2012).

3. Nous prenons en compte tous les motifs d'origine (résidence, travail et activités). On considère que les individus pourraient développer des stratégies pour accéder aux ressources depuis des lieux d'origine autres que leur lieu de résidence. Les individus organiseraient leurs journées à partir des « chaînes d'activités », afin d'optimiser leurs déplacements (Hubert, 2004). 
4. Si des nombreux travaux insistent exclusivement sur l'accès au travail (Wenglenski, 2004), nous nous intéressons aux mobilités hors travail et études, qui peuvent dessiner des formes d'inégalités aussi voire plus significatives. Nous considérons que les mobilités domicile-travail s'inscrivent dans des logiques particulières de mobilité qui méritent d'être étudiées séparément des autres types d'activité.

5. Cet article présente les résultats préliminaires d'une thèse en cours. Au moment de la rédaction les entretiens avaient été réalisés auprès de douze ménages. Cet échantillon exploratoire, modeste, a permis de tracer des résultats significatifs dont on rend ici compte sous forme de transcriptions et de narrations des itinéraires de mobilité, mis en regard de l'analyse des données.

6. Le potentiel d'accès a été originellement décliné en six catégories distinctes $\left(---,--,-^{-,+,++,+++}\right)$ que nous avons regroupé en trois catégories afin de rendre l'analyse plus lisible. Ces catégories ont été discrétisées de la manière suivante : de 0 à 0,25 , de 0,25 à 0,5 , de 0,5 à 1 , de 1 à 5,5 à 20 et plus de 20.

7. Soit 622 Reais en 2012.

8. Les "gains d'accessibilité » sont représentés par les déplacements réalisé depuis des secteurs moins équipés vers des secteurs plus équipés, c'est-à-dire, ceux réalisés entre les secteurs «faible -moyen », « faible-fort » et « moyen -fort ».

\section{ABSTRACTS}

This paper aims at evaluating inequalities in access to urban resources the Metropolitan Region of Belo Horizonte in the light of the recent socio-spatial evolutions of the peripheral spaces. First, the analysis of resources relative density helps identifying their spatial distribution in the MRBH. Furthermore, the exploitation of the latest household-travel survey and a set of qualitative interviews emphasize that, despite the diversification process experienced in the peripheries, the inequalities in access to urban resources are still pronounced. The mobility behavior of the upper classes is shaped by their individual choices, whilst the poorest are still forced to develop strategies to access urban resources.

Cet article a pour but d'analyser les inégalités d'accès aux ressources urbaines des habitants des franges périphériques de la Région Métropolitaine de Belo Horizonte au regard des évolutions socio-spatiales constatées récemment dans ces espaces. Dans un premier temps, l'analyse de la densité relative de ressources permet d'identifier leur distribution dans la RMBH. Ensuite, l'exploration des enquêtes ménages-déplacements et des entretiens semi-directifs permet de déterminer que, malgré la croissante diversification des espaces périphériques, les inégalités d'accès demeurent prononcées. Les populations aisées suivent des logiques de mobilité guidées par leurs choix individuels, tandis que les populations modestes sont toujours contraintes de déployer des stratégies afin d'accéder aux ressources urbaines. 


\section{INDEX}

Mots-clés: mobilité quotidienne, étalement urbain, inégalité spatiale, accessibilité, ressource urbaine

Keywords: daily mobility, urban sprawl, spatial inequality, accessibility, urban resources Subjects: Sur le Champ - Sur le Terrain

\section{AUTHOR}

\section{EUGÊNIA VIANA CERQUEIRA}

Eugênia Viana Cerqueira, eugeniadoria@gmail.com est Doctorante contractuelle à l'Université Paris 1 Panthéon-Sorbonne et membre del'UMR Géographie-Cités, LABEX Dynamite. Elle a publié récemment :

- Viana Cerqueira E., 2014. A evolução das formas de gentrificação : estratégias comerciais locais e o contexto parisiense. Cadernos Métropole, 16, 32, p. 417-436.

- Viana Cerqueira E., 2015. As novas lógicas de fortificação residencial nas periferias metropolitanas de Belo Horizonte : quais impactos sobre a segregação social ? Urbe : Revista de Gestão Urbana, 7, 2, p.195-210.

- Berroir S., Fleury A., Fol S., Guérois M., Vallée J., Viana Cerqueira E. Petites villes dans les franges de l'aire métropolitaine parisienne : entre ancrages locaux et intégration métropolitaine. Colloque Aux frontières de l'urbain. Petites villes du monde : émergence, croissance, rôle économique et social, intégration territoriale, gouvernance, Avignon, 22-24 janvier 2014. 